\title{
Refinement of a 0D Turbulence Model to Predict Tumble and Turbulent Intensity in SI Engines. Part II: Model Concept, Validation and Discussion
}

\author{
Author, co-author (Do NOT enter this information. It will be pulled from participant tab in \\ MyTechZone) \\ Affiliation (Do NOT enter this information. It will be pulled from participant tab in MyTechZone)
}

\begin{abstract}
As known, reliable information about underlying turbulence intensity is a mandatory pre-requisite to predict the burning rate in quasidimensional combustion models. Based on 3D results reported in the companion part I paper, a quasi-dimensional turbulence model, embedded under the form of "user routine" in the GT-Power" software, is here presented in detail. A deep discussion on the model concept is reported, compared to the alternative approaches available in the current literature. The model has the potential to estimate the impact of some geometrical parameters, such as the intake runner orientation, the compression ratio, or the bore-to-stroke ratio, thus opening the possibility to relate the burning rate to the engine architecture.
\end{abstract}

Preliminarily, a well-assessed approach, embedded in GT-Power commercial software v.2016, is utilized to reproduce turbulence characteristics of a VVA engine. This test showed that the model fails to predict tumble intensity for particular valve strategies, such LIVC, thus justifying the need for additional refinements.

The model proposed in this work is conceived to solve 3 balance equations, for mean flow kinetic energy, tumble vortex momentum, and turbulent kinetic energy (3-eq. concept). An extended formulation is also proposed, which includes a fourth equation for the dissipation rate, allowing to forecast the integral length scale (4-eq. concept).

The impact of the model constants is parametrically analyzed in a first step, and a tuning procedure is advised. Then, a comparison between the 3 - and the 4-eq. concepts is performed, highlighting the advantages of the 3-eq. version, in terms of prediction accuracy of turbulence speed-up at the end of the compression stroke. An extensive 3 -eq. model validation is then realized according to different valve strategies and engine speeds.

The user-model is then utilized to foresee the effects of main geometrical parameters analyzed in part I, namely the intake runner orientation, the compression ratio, and the bore-to-stroke ratio. A two-valve per cylinder engine is also considered. Temporal evolutions of 0D- and 3D-derived mean flow velocity, turbulent intensity, and tumble velocity present very good agreements for each investigated engine geometry and operating condition. The model, particularly, exhibits the capability to accurately predict the tumble trends by varying some geometrical parameter of the engine, which is helpful to estimate the related impact on the burning rate.

Summarizing, the developed 0D model well estimates the in-cylinder turbulence characteristics, without requiring any tuning constants adjustment with engine speed and valve strategy. In addition, it demonstrates the capability to properly take into account the intake duct orientation and the compression ratio without tuning adjustments. Some minor tuning variation allows predicting the effects of bore-to-stroke ratio, as well. Finally, the model is verified to furnish good agreements also for a two-valve per cylinder engine, and with reference to two different high-performance engines.

\section{Introduction}

In the concept and design of modern Internal Combustion Engines (ICEs), simulation tools play an increasing role, with the aim of reducing the experimental activity need and the related costs and time. Among the available modeling approaches, 1D simulation proved to properly describe ICE performance, mainly depending on the sub-models' reliability of in-cylinder phenomena, namely turbulence, combustion, knock, cyclic dispersion, and heat transfer. More refined phenomenological models, if compared to the empirical approaches, allow to provide information also in absence of experimental data, thanks to their improved predictive capability.

Among the above listed in-cylinder phenomena, a proper description of the turbulence represents the most relevant prerequisite to forecast the combustion evolution in Spark-Ignition (SI) ICEs. It is broadly recognized that flame front propagation is supported by the turbulence within the combustion chamber. The mechanisms leading to flame front enhancement are explained by different theories, such as eddy burn-up model [1-3], flame surface density approach [4], and fractal model $[5,6]$. Whichever is the employed approach, results are always strictly dependent on the reliability of the turbulence submodel coupled to the combustion one.

It is widely accepted that turbulence level close to the compression TDC, namely in the most relevant cycle portion for combustion development, is determined by the collapse of ordered flow structures, produced during the intake phase. Concerning SI ICEs, the most important flow structure, for an effective turbulence production, proved to be the tumble motion. The latter is strictly determined by the intake port design, which, however, has to provide an adequate cylinder filling, too. Tumble development is also affected by the intake valve strategy, as shown in $[7,8]$. Other constructive parameters, such as the Compression Ratio (CR) or the Bore-toStroke (BS) ratio, play a role in the tumble production and in the turbulence intensity during the combustion.

Engine architectures with four-valve per cylinder proved to more favorably promote a single ordered tumble vortex, while a two-valve design usually splits the kinetic energy of the intake flow in 
secondary ordered flow motions, which only partially contribute to turbulence production.

A reliable and predictive turbulence model has to be able to describe variations in both operating conditions and the engine architecture. On the other hand, the development of such a OD model represents a very challenging task, since it aims to synthesize very complex $3 \mathrm{D}$ phenomena.

The current literature presents various approaches to face the above issue, most of them classified as $k-\varepsilon$ and $K-k$ models. Original proposals of $k-\varepsilon$ models are reported in [9] and [10]. In those works, $0 \mathrm{D}$ equations of turbulent kinetic energy, $k$, and dissipation rate, $\varepsilon$, are derived from 3D turbulence models. A first attempt to take into account the contribution to turbulence from ordered flow structures is proposed in [11], where source terms, related to swirl and squish motions, are introduced. A more recent version of such approach is proposed in [12]. A different method consists in describing the energy cascade from mean flow kinetic energy, $K$, into turbulent kinetic energy, $k$, usually referred as $K-k$ models $[13,14]$. Turbulence dissipation is, hence, derived from the integral length scale, $L_{I}$. The evolution of $L_{I}$ during the engine cycle is commonly related to the instantaneous cylinder volume, piston-head distance, or reconstructed by mathematical functions $[13,14]$. Such a choice is supported by the observation that the integral length scale evolution does not significantly change with the operating conditions.

A refinement of a $K-k$ approach involves a more direct description of the ordered flow motions, instead of the overall in-cylinder mean flow velocity. A simplified methodology is proposed in [15], where a linear decrease of the tumble vortex is imposed as a function of the piston distance from the cylinder head. In [16,17], more physical tumble models are introduced, capable of considering the geometry of the intake system, and the valve and the cylinder port inclination. In [18], a model is developed, describing both tumble and swirl motions, and their dissipation in turbulence. A critical issue in such methodologies is the description of the flow structure decay during the compression stroke, due to the shear stresses. 3D CFD studies showed that the decay rate well correlates with the piston-head distance. For this reason, the above phenomenon is described by a decay function depending on the piston position normalized by the bore [18]. A more comprehensive approach is proposed in [19], where $K-k$ and $k-\varepsilon$ models are synthesized, leading to the so-called $K$ $k-\varepsilon$ model. Such methodology takes into account the energy cascade mechanism typical of $K-k$ model, and directly describes the turbulence dissipation. In addition, a tumble model is provided by a dedicated additional equation. Tumble decay directly affects the turbulence level close-to-TDC. The model proves a good accuracy in predicting both tumble ratio and turbulence intensity under various operating conditions, valve strategy and engines. Some inaccuracy appears in describing operations with delayed intake valve closure. This is due to its poor ability in capturing the backflow effects through the intake valve, which partially degrades the main tumble vortex.

Based on the above literature overview, phenomenological models throughout time have reached an improved predictive capability. In the past, models were commonly validated in an indirect way comparing numerical and experimental burn rates $[3,20]$. The availability of experimental data about mean flow and/or turbulence fields for model validation purpose was still rather limited. In addition, most of the available experimental data on tumble and turbulence were based on measuring techniques which allowed to observe only a limited portion of the cylinder. These were suitable for validating a 3D model [21], where the velocities could be calculated at different spatial locations inside the cylinder. In phenomenological

Page 2 of 12 models, where spatial homogeneity of thermodynamic properties and fluid dynamic variables are assumed, the use of location-specific experimental data for validation purpose is rather questionable. However, even more recent and advanced dynamic flow visualization techniques, allowing for the investigation of the tumble flow field, are limited to a very reduced set of operating conditions [22], because of their high costs and complexity.

The most robust approach for phenomenological model validation is hence based on the 3D CFD results, which easily include main fluiddynamic phenomena occurring inside the combustion chamber. In addition, during time, 3D simulations are becoming more and more accurate and reliable, thanks to the adoption of more refined numerical approaches, and to the growing computing power. 3D codes, moreover, allow to investigate various operating conditions and engine geometries more easily than experimental techniques. Phenomenological model validation, based on averaged 3D results, has been successfully followed in $[13,16,18,19]$.

Available turbulence models, however, still present some limitations. In fact, some of them [19] mainly focus on the capability of reproducing the engine operating parameters, trying to synthesize geometrical features into a number of tuning constants. On the other hand, other models, directly taking into account the geometry, lack of an extensive validation [17] or fail in describing some geometrical modifications [16]. Based on the authors' knowledge, in the current literature, a model able to simultaneously and properly reproduce both engine operating parameters, including intake valve strategy (both EIVC, conventional Full Lift - FL, and LIVC) and engine geometrical parameters, is still missing. The main aim of this work is its achieving, where 3D results presented in companion part I paper [23] are employed to refine and validate a model previously developed by the authors [24]. The model represents an extension of a conventional two-equation $K-k$ formulation, where tumble and dissipation rate equations are also added (4-eq. concept). A new formulation for the turbulent energy production is also proposed, based on the analyses of 3D data. A variant of the 4-eq. model is besides conceived, where the $\varepsilon$ equation is substituted by a predefined reconstruction of the integral length scale (3-eq. concept). Both are implemented in GT-Power ${ }^{\mathrm{TM}}$ environment under the form of user routine.

The paper is organized as follows: first, the investigated operating conditions and the analyzed engine arrangements are introduced. In a second stage, the outcomes of a reference state-of-art model, the one embedded in the standard version of GT-Power ${ }^{\mathrm{TM}}$, is discussed, highlighting some limitations under unconventional valve strategies. Then, the model equations and assumptions are presented. An assessment between the 3- and 4-eq. versions of the model is proposed. Finally, the model accuracy is extensively proved under various operating conditions and engine geometries.

\section{Description of tested operating conditions and engine configurations}

In order to develop and validate the proposed model, various 3D simulations are carried out, with reference to two different engines, labelled as Engine A and Engine B. The former is an in-series turbocharged downsized engine, equipped with 4 valves per cylinder and a VVA system on the intake side. This system, based on an electro-hydraulic VVA device, allows to flexibly control the closure angle, so to realize both EIVC and LIVC strategies. Various operating points are investigated, with different rotational speeds (1500 to $5500 \mathrm{rpm}$ ) and valve strategies, including both EIVC, FL and LIVC. Representative valve lift profiles are plotted in Figure 1. 


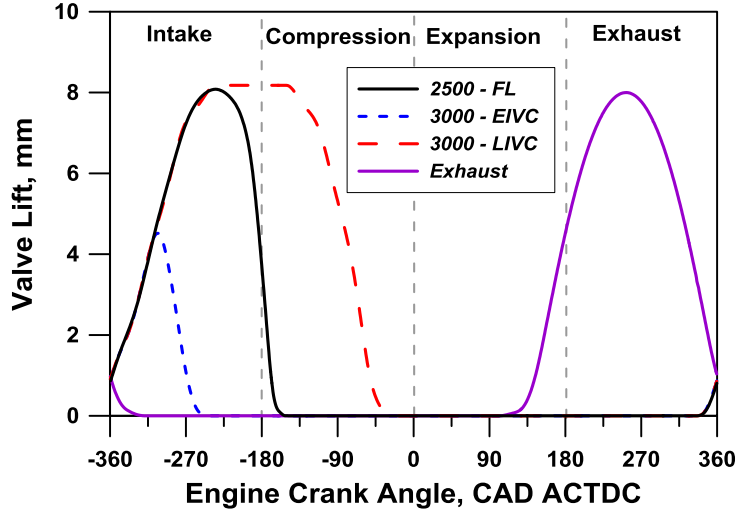

Figure 1. Exhaust lift and representative intake valve lift profile for EIVC, Full Lift and LIVC strategies.

The Engine B is not a commercial engine, but just represents a framework "virtually" modified to investigate the effects of architectural and geometrical adjustments. It exemplifies a naturally aspirated engine, equipped with 4 valves per cylinder, characterized by a low BS ratio and a fixed valve strategy. Starting from the base architecture (labelled as "Low Tumble" in the following), an increase in the intake duct bending is applied up to 15 degrees, corresponding to a "tumble promoting" design (labelled as "High Tumble").

Additional analyses are carried out with different compression ratios. A modified version of the Engine B, scaled to realize a high BS design, is simulated, too. Finally, a 2-valve per cylinder unit and two commercially available high-performance engines, are analyzed.

\section{Comparison with GT-Power Turbulence Model}

Version 2016 of commercial software GT-Power includes one of most recent approaches for turbulence modeling. The procedure is based on the Fogla et al. [19] work. The reliability of this model is tested with reference to the $3 \mathrm{D}$ results obtained on the Engine A, for different valve strategies and engine speeds. According to the discussion on the averaging technique of part I paper, target 3D data refer to mass-averaged quantities of mean flow velocity, turbulent kinetic energy, and integral length scale, while turbulent intensity and dissipation rate are derived from the above quantities combination.

The model is preliminary tuned with reference to FL engine operation, typical of high load conditions. The values of four "multipliers" acting on an intake related mean flow production, turbulence production from mean flow, length scale, and tumble decay intensity, are identified to reach a good matching with $3 \mathrm{D}$ findings. The model also requires as an input the steady tumble coefficient, function of the valve lift, which is not available for the considered engine. A profile derived from available data in current literature is specified [7], which may affect the result quality. At the same time, this issue represents a limitation of the approach, since the above datum is often unavailable for the modelers. Nevertheless, an adequate agreement is reached on main turbulence parameters, as confirmed by Figure 2. Notably, the model exhibits the ability to reproduce the correct trends of dissipation rate, integral length scale, tumble ratio, and turbulence intensity, especially during intake and compression strokes. However, Figure $2 \mathrm{c}$ shows that in the 0D approach, the tumble ratio rapidly increases as soon as the compression stroke begins, while 3D results present an initial decay. This is due to the decay function formulation employed in [19], assuming a null value at BDC. Therefore, the tumble ratio is slightly overestimated. The same multipliers' set is applied to estimate turbulence data for EIVC and LIVC (Figure 3 and Figure 4, respectively). In these cases, the model shows some more relevant

Page 3 of 12 inaccuracies, since the tumble ratio is underestimated for the EIVC strategy (Figure 3c), while it is highly overestimated for the LIVC one (Figure 4c). Consequently, the turbulence speed-up towards the compression stroke end is also incorrectly predicted, especially for the late strategy (Figure 4d). These limitations, also recognized by the model authors [19], justify the need of a model refinement.
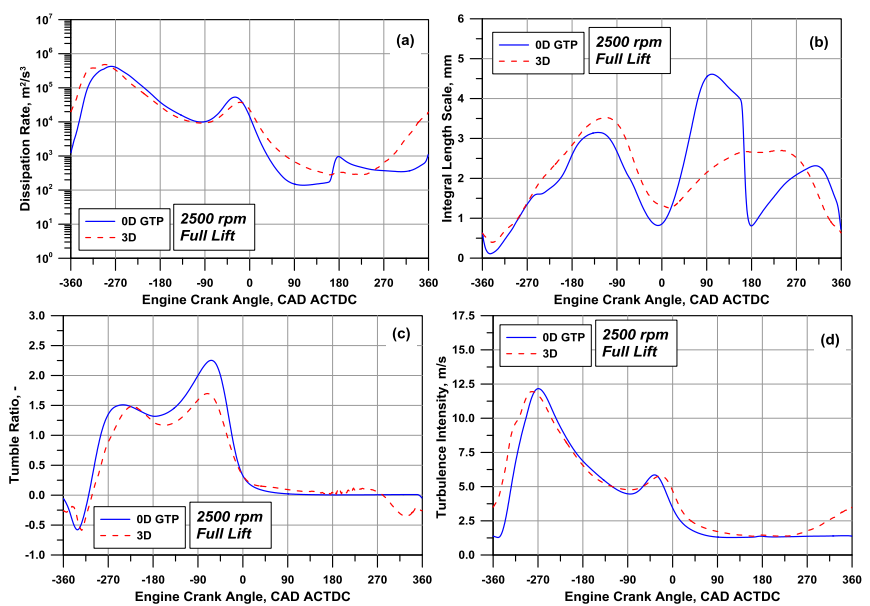

Figure 2. Comparison of standard GT-Power model with 3D results. Full Lift.
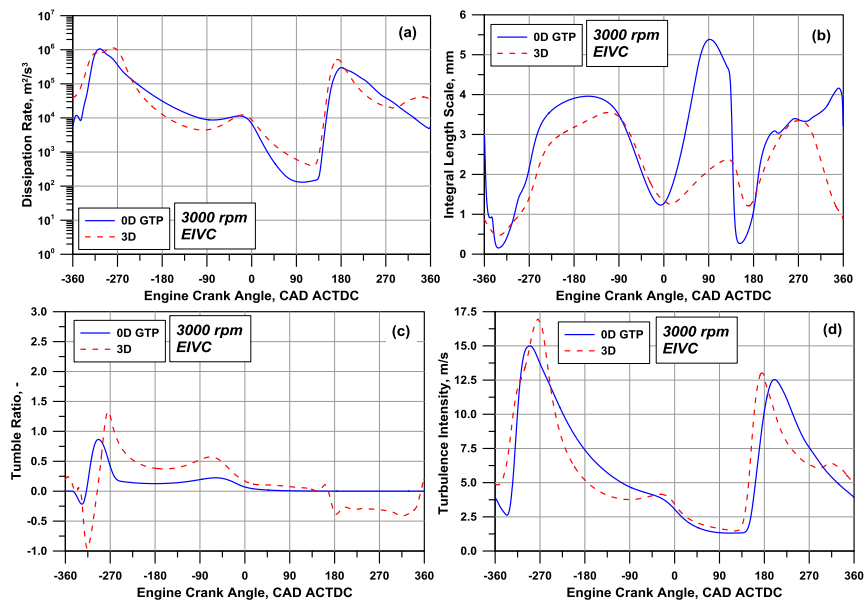

Figure 3. Comparison of standard GT-Power model with 3D results. Early IVC.
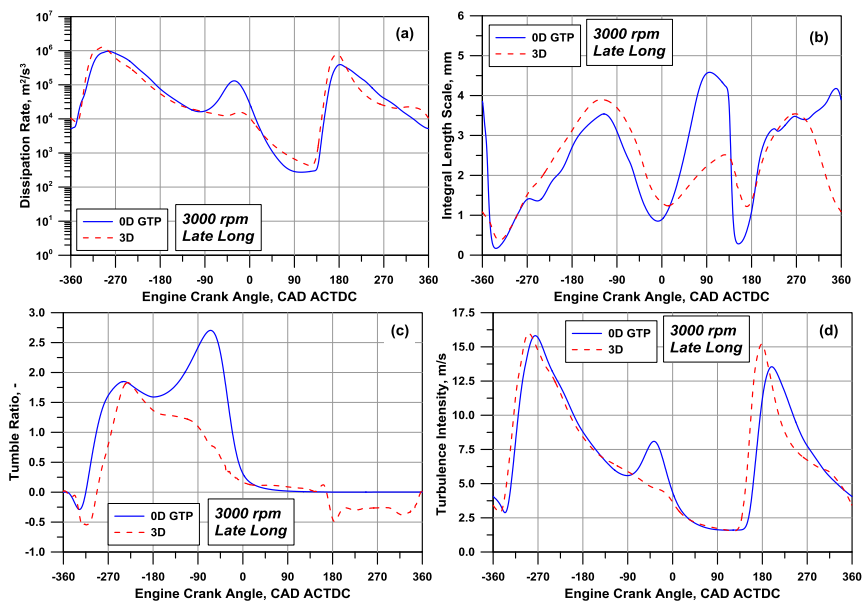

Figure 4. Comparison of standard GT-Power model with 3D results. Late IVC. 


\section{D Model Concept}

The proposed 0D model derives from 3D RNG $k$ - $\varepsilon$ turbulence formulation, presented in part I. To synthesize the model in a OD framework, the assumptions and relations below are utilized:

$$
\begin{gathered}
\frac{\partial u_{i}}{\partial x_{i}}=\text { velocity divergence } \approx-\frac{\dot{\rho}}{\rho} \\
P=2 m v_{t}\left(S_{i j} S_{i j}\right) \text { to be modelled } \\
v_{t}=c_{\mu} \frac{k^{2}}{\varepsilon} \quad g_{i} \approx 0 \quad \frac{\partial^{2}}{\partial x^{2}} \approx 0 \\
\frac{d m k}{d t}=(\dot{m} k)_{\text {inc }}-(\dot{m} k)_{\text {out }}+\frac{2}{3} \frac{\dot{\rho}}{\rho}\left(-m v_{t} \frac{\dot{\rho}}{\rho}+m k\right)+P-m \varepsilon \\
\frac{d m \varepsilon}{d t}=(\dot{m \varepsilon})_{\text {inc }}-(\dot{m} \varepsilon)_{\text {out }}+c_{\varepsilon 1} \frac{\varepsilon}{k}\left[P-\frac{2}{3} m v_{t}\left(\frac{\dot{\rho}}{\rho}\right)^{2}+\frac{2}{3} m k \frac{\dot{\rho}}{\rho}\right] \\
-c_{\varepsilon 2} \frac{m \varepsilon^{2}}{k}-c_{\varepsilon 4} m \varepsilon \frac{\dot{\rho}}{\rho}-\frac{c_{\mu} \eta^{3}\left(1-\frac{\eta}{\eta_{0}}\right)}{1+\beta \eta^{3}} \frac{m \varepsilon^{2}}{k}
\end{gathered}
$$

According to definitions in part I, the parameter $\eta$ is expressed as:

$$
\eta=S \frac{k}{\varepsilon}=\sqrt{\frac{P}{m v_{t}}} \frac{k}{\varepsilon}
$$

Constants in eq. (1) and (2) are the same ones specified in the 3D model. Two additional equations have to be formulated to describe the time evolution of the specific angular momentum associated to the tumble motion, and the mean flow kinetic energy, defined as:

$$
T=U_{T} r_{T} \quad K=\frac{U_{f K}^{2}}{2}
$$

$U_{f K}$ and $U_{T}$ being the mean flow and tumble vortex velocities, and $r_{T}$ being the related tumble radius. To be more precise, $K$ includes contributions of both unorganized and organized flows developing inside the cylinder. Based on the above definitions, angular momentum and kinetic energy balance equations look like:

$$
\begin{aligned}
& \frac{d m T}{d t}=(\dot{m} T)_{\text {inc }}-(\dot{m} T)_{\text {out }}-f_{d} \frac{m T}{t_{T}} \\
& \frac{d m K}{d t}=(\dot{m} K)_{\text {inc }}-(\dot{m} K)_{\text {out }}-f_{d} \frac{m K}{t_{T}}+m K \frac{\dot{\rho}}{\rho}-P
\end{aligned}
$$

First and second term in both equations describe incoming and outcoming convective flows through the valves, respectively, while the third one expresses the decay due to the shear stresses with combustion chamber walls. To model this effect, a decay function, $f_{d}$, and a characteristic time scale, $t_{T}$, are introduced:

$f_{d}=c_{f d 0}+c_{f d m}\left[\max \left(\frac{B}{H}, 1\right)-1\right]$

$t_{T}=\frac{r_{T}}{u^{\prime}}$
The decay function only depends on instantaneous geometrical dimensions of the combustion chamber (see sketch in Figure 5). It is built to be particularly high near TDC, where tumble vortex collapse is expected to occur. Parameters $c_{f d 0}$ and $c_{f d m}$ are tuning constants: the former is active all over the engine cycle, and represents an offset introduced to consider tumble and kinetic energy dissipation due to internal viscous forces. The above offset allows to improve the tumble ratio trend prediction around the BDC. $c_{f d m}$ parameter is a multiplier utilized to adjust the intensity of tumble collapse.

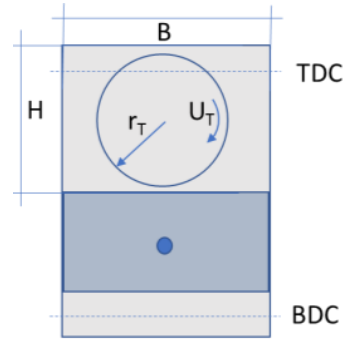

Figure 5. Qualitative sketch of the tumble vortex.

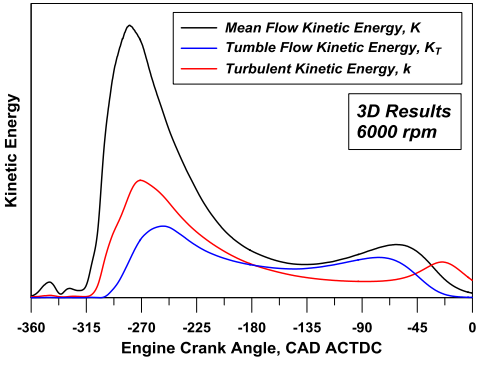

Figure 6. Kinetic energies associated to mean, tumble and turbulent flows.
Coherently with $k$ formulation, $K$ equation includes an additive compressibility term, $m K \dot{\rho} / \rho$, and a subtractive turbulent production term, $P$, expressing the energy cascade mechanism. The latter quantity, related to the strain tensor rate, $S_{i j}$, requires some modeling assumptions. Basically, the key conjecture is that the difference between overall mean flow kinetic energy, $K$, and the one associated to the tumble motion, $K_{T}=U_{T}^{2} / 2$, is the main driver for turbulence production. In fact, while $K$ is associated to both organized and disordered flow structures, $K_{T}$ only considers ordered kinetic energy. This idea is supported by Figure 6, plotting 3D-derived profiles of above quantities, together with turbulent kinetic energy, $k$. During the first part of the intake stroke, mean flow kinetic energy is much higher than tumble associated kinetic energy. This is because of the interaction between the incoming flow and the piston, which does not allow the development of an ordered flow structure. In this phase, turbulent kinetic energy is highly produced. Later, an intense tumble motion arises, due to the high intake valve lift and piston position far from the cylinder head. Starting from this phase, turbulence production reduces, until the tumble motion collapses, and turbulence is once again created. Based on above discussion, energy cascade term, $P$, is modeled as:

$$
P=c_{P K k} m \frac{K-K_{T}}{t_{T}}
$$

$c_{P K k}$ being a model constant, modulating the energy transfer from mean flow to turbulent one.

Concerning the convective terms in eqs. (1)-(4), the following relations are utilized, holding cylinder incoming and outcoming contributions:

$(\dot{m} k)_{i n c}=0$

$(\dot{m \varepsilon})_{i n c}=\frac{(\dot{m} K)_{i n c}}{t_{T}} \frac{k}{K}$

$(\dot{m} T)_{i n c}=r_{T}\left(\dot{m}_{i n f} c_{T i n 0} v_{T i n f}-\dot{m}_{e x f} v_{T e x f}-\dot{m}_{e x b} v_{T e x b}\right)$ 


$$
\begin{aligned}
& (\dot{m} k)_{\text {out }}=k\left(\dot{m}_{\text {inb }}+\dot{m}_{\text {exf }}\right) \\
& (\dot{m} \varepsilon)_{\text {out }}=\varepsilon\left(\dot{m}_{\text {inb }}+\dot{m}_{\text {exf }}\right) \\
& (\dot{m} T)_{\text {out }}=2 T\left(\dot{m}_{\text {inb }}+\dot{m}_{\text {exf }}\right) \\
& (\dot{m} K)_{\text {out }}=K\left(\dot{m}_{\text {inb }}+\dot{m}_{\text {exf }}\right)
\end{aligned}
$$$$
(\dot{m} K)_{i n c}=\frac{1}{2}\left[\dot{m}_{\text {inf }}\left(c_{K i n 0} v_{\text {Kinf }}\right)^{2}+\dot{m}_{\text {exf }} v_{\text {Kexf }}^{2}+\dot{m}_{\text {exb }} v_{K e x b}^{2}\right]
$$

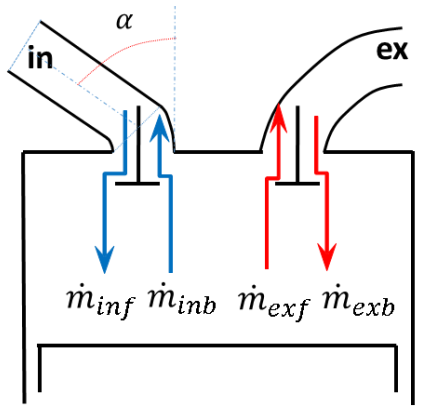

Figure 7. Definition of $\alpha$ angle and of forward and reverse mass flow rates.

In equations (12) and (13), velocities $v_{T}$ and $v_{K}$ include flow losses through the valves. More precisely, they comprise tumble and discharge coefficients, respectively. Those coefficients are automatically specified as a function of the valve lift. They also vary with the $\alpha$ angle between the intake runner and the cylinder axis (Figure 7). This last dependency is introduced, at this research stage, only for the tumble coefficient. The possibility to tune the above coefficients is offered by global multipliers $c_{\text {Tin } 0}$ and $c_{\text {Kin } 0}$.

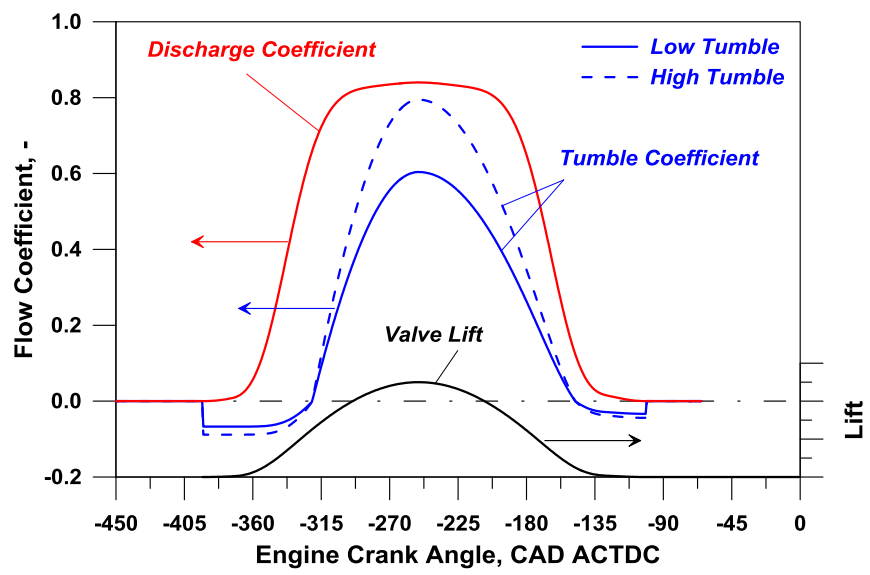

Figure 8. Internally computed discharge and tumble coefficients.

Figure 8 shows an example of flow coefficients obtained for the intake valve. The developed procedure is based on $3 \mathrm{D}$ results presented in part I, and specifically on the analysis of velocity distribution around the intake valve (Figure 23 in Part I). A reduced negative tumble coefficient is specified at low valve lift (reverse tumble), due to the piston and the cylinder wall flow interaction. Later during the intake stroke, a positive tumble flow develops, whose intensity highly depends on the $\alpha$ angle. The automatic estimation of flow coefficients allows to overcome one of the limitations showed by other approaches available in the literature, requiring, as said, the availability of information from flow benches. Validation of the estimated coefficients is currently ongoing by Page 5 of 12 steady state $3 \mathrm{D}$ simulations. An indirect validation is, however, given in the following, in terms of predicted tumble levels by varying the runner inclination.

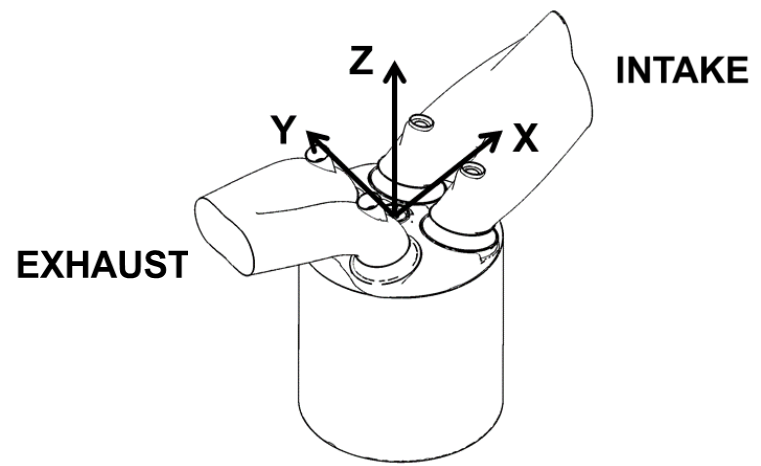

Figure 9. Definition of the reference axes

Although less important, last two terms of eq. (12) give a subtractive contribution to tumble intensity, assuming that the exhaust flow, both in forward and reverse direction, produces a reverse tumble, opposite to the conventional positive orientation defined in Figure 5. Similar considerations justify the "2" multiplier in eq. (16): a reverse intake flow is observed to substantially destroy the tumble vortices inside the cylinder (see for example Figure 15 of Part I). The system of eqs. (1)-(4) constitutes a "4-eq. model concept"; once integrated, it allows to derive the integral length scale as:

$$
L_{I}=C_{\mu}^{3 / 4} \frac{k^{3 / 2}}{\varepsilon}
$$

3D analyses showed that the above parameter, mass-averaged over the whole 3D domain, is almost independent on the engine operating conditions. For this reason, a single profile can be specified once for a selected geometry, based on predefined shape functions, as described in [24]. In this case, eq. (2) can be neglected and substituted by eq. (18), inverted to derive the dissipation rate ("3-eq. model concept"):

$$
\varepsilon=C_{\mu}^{3 / 4} \frac{k^{3 / 2}}{L_{I}}
$$

Similarly, a characteristic tumble radius around y-axis (see Figure 9 for cylinder axes definition), $r_{T}$, can be derived from $3 \mathrm{D}$ analyses, here defined as:

$$
r_{T}=\frac{\sum_{c y l} m_{i}\left[\left(x_{i}-x_{G}\right)^{2}+\left(z_{i}-z_{G}\right)^{2}\right]}{\sum_{c y l} m_{i} \sqrt{\left(x_{i}-x_{G}\right)^{2}+\left(z_{i}-z_{G}\right)^{2}}}
$$

where $m_{i}$ is the mass in the $i$-th cell, $x_{i}, y_{i}, z_{i}$ are the Cartesian coordinates of the $i$-th cell center, and $x_{G}, y_{G}, z_{G}$ are the Cartesian coordinates of the in-cylinder mass center. Alternatively, a CAD procedure can provide the same parameter by processing the actual $3 \mathrm{D}$ geometry of the engine under investigation. Simpler approaches for $r_{T}$ calculation are based on the analytical relations proposed in [18]. The $r_{T}$ formulation here employed is very similar to the one reported in [19]:

$$
r_{T}=c_{r T 0}+c_{r T m} \frac{1}{4} \sqrt{B^{2}+H^{2}}
$$

$\mathrm{c}_{r T 0}$ and $c_{r T m}$ being two adjustable parameters. 
Figure 10 puts into evidence the possibility to specify a single integral length scale profile and a single value for parameters $\mathrm{c}_{r T 0}$ and $c_{r T m}$ to properly fit $3 \mathrm{D}$ derived trends of both $L_{I}$ and $r_{T}$, at varying the engine speed and the valve strategy. Integral length scale disagreement along the exhaust stroke does not influence at all the turbulence levels during intake, compression and expansion phases. It must be emphasized that parameters required to define the shape functions of integral length scale and tumble radius cannot be considered as tuning constants, since they do not depend on operating conditions and can be univocally identified based on 3D results.
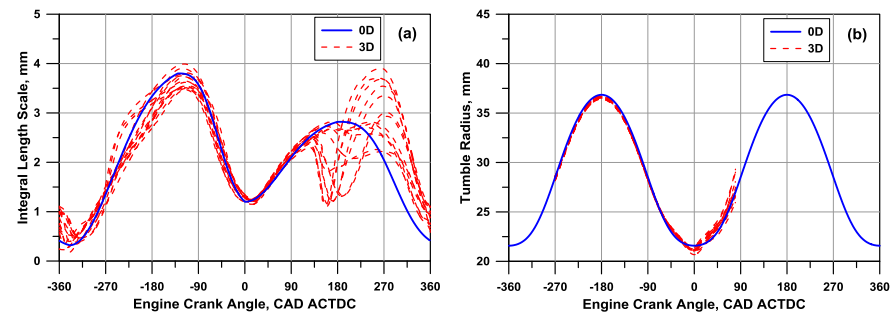

Figure 10. Geometrical invariants: (a) Integral length scale, (b) tumble radius.

\section{Model tuning}

The turbulence model includes 5 tuning constants, namely:

- $\quad c_{K i n 0}$, acting on mean flow production during intake stroke;

- $\quad c_{\text {Tin } 0}$, acting on tumble production during intake stroke;

- $\quad c_{f d 0}$, defining the offset of the decay function;

- $\quad c_{f d m}$, adjusting the intensity of tumble collapse near TDC;

- $\quad c_{P K k}$, adjusting the turbulence production from mean flow.
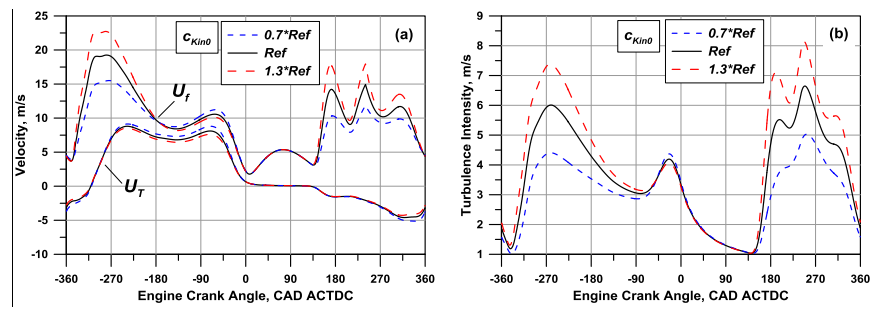

Figure $11 . c_{\text {Kin } 0}$ effect on mean flow, tumble and turbulence intensity.
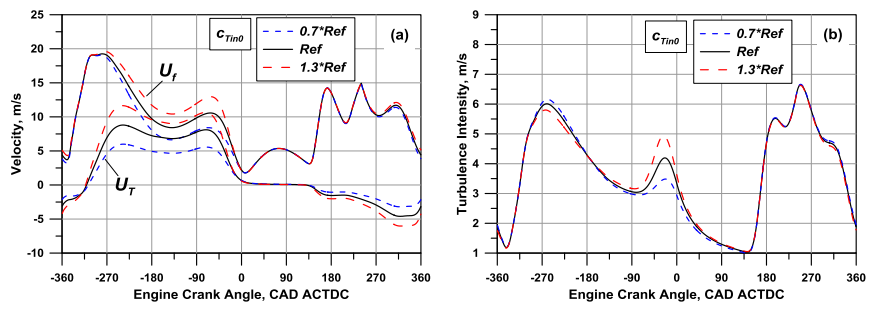

Figure $12 . c_{\text {Tin } 0}$ effect on mean flow, tumble and turbulence intensity.
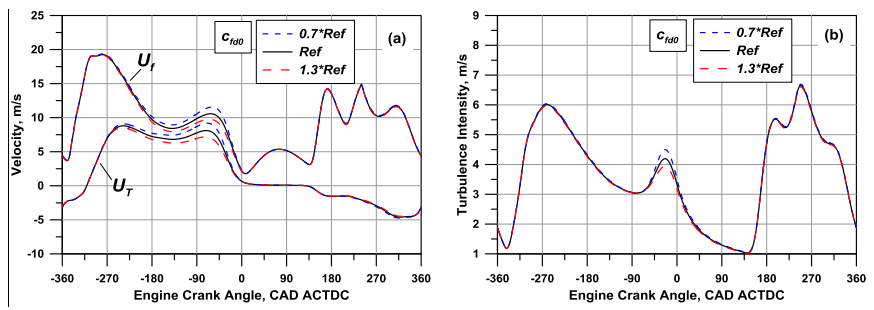

Figure 13. $c_{f d 0}$ effect on mean flow, tumble and turbulence intensity.

Page 6 of 12
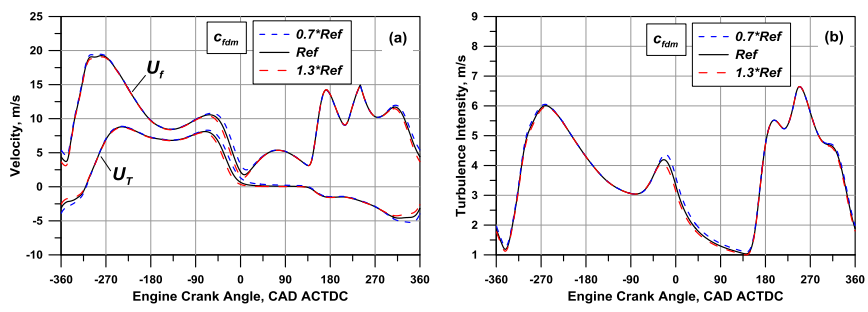

Figure 14. $c_{f d m}$ effect on mean flow, tumble and turbulence intensity.
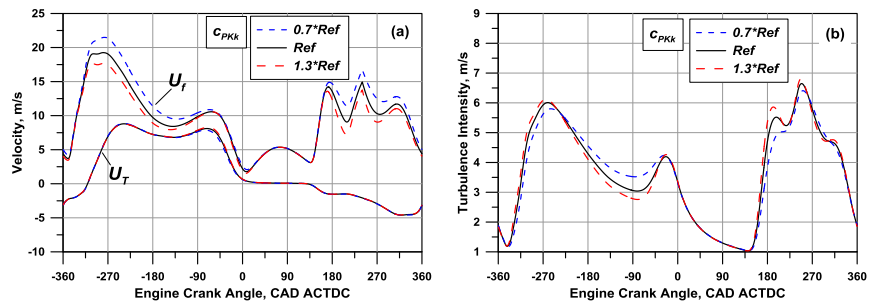

Figure 15. $c_{p K k}$ effect on mean flow, tumble and turbulence intensity.

Figure 11 to Figure 15 depict the effect of each tuning constant, by varying a standard reference value of $\pm 30 \%$. The impact on mean flow and tumble velocities is highlighted on the left figures, while turbulence intensity variations are plotted on the right ones. To have a more consistent comparison with 3D data, mean flow velocity takes also into account the kinetic energy provided by piston motion, according to the relations:

$U_{f}=\sqrt{2\left(K+K_{\text {pist }}\right)} \quad K_{\text {pist }}=\frac{1}{2} \frac{u_{\text {pist }}^{2}}{3}$

Figure 11 shows that $c_{\text {Kin } 0}$ constant significantly modifies the mean flow and turbulence peaks in the middle of the intake stroke, which however turns in a reduced modification of the turbulence speed-up close to TDC. On the contrary, $c_{\text {Tin } 0}$ variation in Figure 12 has a considerable impact on each monitored parameter, such as $U_{f}, U_{T}$ and $u^{\prime}$. Actually, a higher tumble level at the compression stroke end determines a greater mean flow velocity. Moreover, the collapse of the more intense tumble motion turns in an increased turbulence speed-up. Figure 13 highlights that, as expected, an increased (reduced) decay function offset, $c_{f d 0}$, promotes (lowers) the decay of both mean flow and tumble velocities, turning in more (less) intense turbulence production close to TDC. The role of the $c_{f d m}$ parameter is, indeed, shown in Figure 14. It underlines that $c_{f d m}$ controls the crank angle for tumble collapse, with minor impact on turbulence peaks. Finally, in Figure 15, the effect of $c_{P K k}$ is illustrated: this constant does not affect the tumble levels, while it modifies turbulence trend during compression stroke. It can be noted that a higher $c_{P K k}$ determines a lower turbulence during compression, due to lower $U_{f}$, although similar $u^{\prime}$ peaks are reached.

Under the above discussed sensitivity analysis, a tuning procedure can be advised. The most important tuning constant appears to be $c_{\text {Tin } 0}$ : its value is adjusted to gain a good matching with $3 \mathrm{D}$-derived tumble level and turbulence speed-up during the compression stroke. Secondly, $c_{K i n 0}$ is identified to reproduce the $3 \mathrm{D}$ mean flow velocity peak. Tumble collapse and close-to-TDC turbulence peak can be further handled by $c_{f d 0}$ and $c_{f d m}$ constants. Finally, the $c_{P K k}$ multiplier is, if needed, fine-tuned to adjust the mean flow and turbulence trends, without any significant impact on tumble levels and turbulence speed-up. 


\section{Comparison of 3-eq. concept vs 4-eq. concept}

Before extensively validating the model, a comparison between the defined 3-eq. and 4-eq. concepts is presented. Test case refers to the Engine A operated at $5500 \mathrm{rpm}$ with FL and LIVC valve strategies (Figure 16 and Figure 17). The results are compared in terms of dissipation rate (a), integral length scale (b), and turbulence intensity (c).

First of all, although the proposed balance equation for the dissipation rate, $\varepsilon$, is fully consistent with the $3 \mathrm{D}$ formulation, its direct integration in the 4eq. concept does not seem to substantially improve the agreement with the 3D-derived trend, compared to the 3eq. model. As already pointed out, a secondary aim of the 4 eq. concept is the possibility to directly recover the evolution of the integral length scale, without the need to assign it by means of predefined shape functions. Nevertheless, the $4 e q$. predicted integral length scale (Figure 16b and Figure 17b) lacks in accurately reproducing the 3D trend during intake and compression strokes, especially for the LIVC case. This behavior can be attributed to the difficulties in predicting the dissipation rate in a $0 \mathrm{D}$ approach.

The dissipation rate, indeed, exhibits spatial not-uniformities within the cylinder, with values close to valves 2 orders of magnitude higher than inside the combustion chamber (see Figure 7 in part I paper). 4eq. model, moreover, does not improve the estimation of the turbulence intensity, as shown in Figure 16c and Figure 17c. For the above reasons, the 3 eq. concept is preferable in terms of simplicity and accuracy. Minor differences arise between the two concepts on other parameters, such as the mean flow and tumble velocities, not reported for brevity. The described behavior is verified to occur in other cases, as well. In the following, the 3eq. concept is hence selected for the model validation.
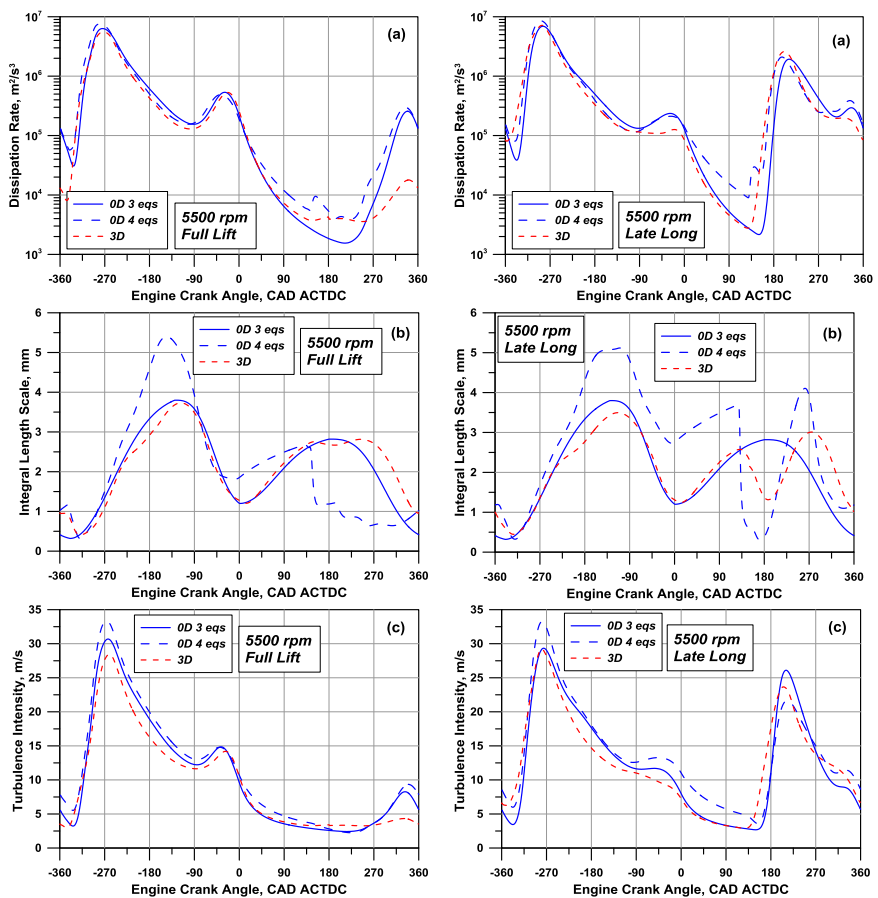

Figure 16. Comparison between 3eq. and 4eq. model concepts. 5500 rpm, FL valve strategy.

\section{Model Validation}

In this paragraph, an extensive model validation is presented. Firstly, the Engine A is considered to verify the model potential in handling very different intake valve control strategies. Then, the Engine B is studied, where some geometrical parameters are virtually changed one by one, namely intake port orientation, compression ratio, and bore-to-stroke ratio. Finally, a two-valve engine and two highperformance engines are considered.

\section{Valve strategy}

Figure 18 to Figure 21 highlight the 0D model consistency for different valve strategies, namely Full Lift, early closure, and late closure. The latter strategy, involving a considerable backward flow through the intake valve, is the most challenging test, since the backflow induces the onset of secondary reverse tumble vortices. For this reason, two different late closures are considered, labelled as "Late Short" and "Late Long" in Figure 20 and Figure 21, where the closure of the intake valve is retarded more and more. It must be stressed that the 5 tuning constants of the model remain unchanged with the engine speed and the valve strategy. The overall model behavior is quite satisfactory, since most of the analyzed operating conditions are reproduced with relevant accuracy. Mean flow velocity, tumble velocity, and turbulent intensity denote a very good agreement with 3D profiles, during each phase of the engine cycle, including the exhaust stroke. Negative values of tumble velocity are also handled with satisfactory accuracy in Figure $18 \mathrm{~b}$ to Figure $21 \mathrm{~b}$. OD model exhibits a lower precision just in one case, that is the tumble velocity for the EIVC case at $5500 \mathrm{rpm}$ (Figure 19b). LIVC cases present a slight overestimation of the turbulence speed-up, while tumble evolution is correctly foreseen. The comparison with GT-Power results in Figure 4, referring to $3000 \mathrm{rpm}$ Late Long strategy, highlights the better accuracy of the model here proposed (Figure 20).
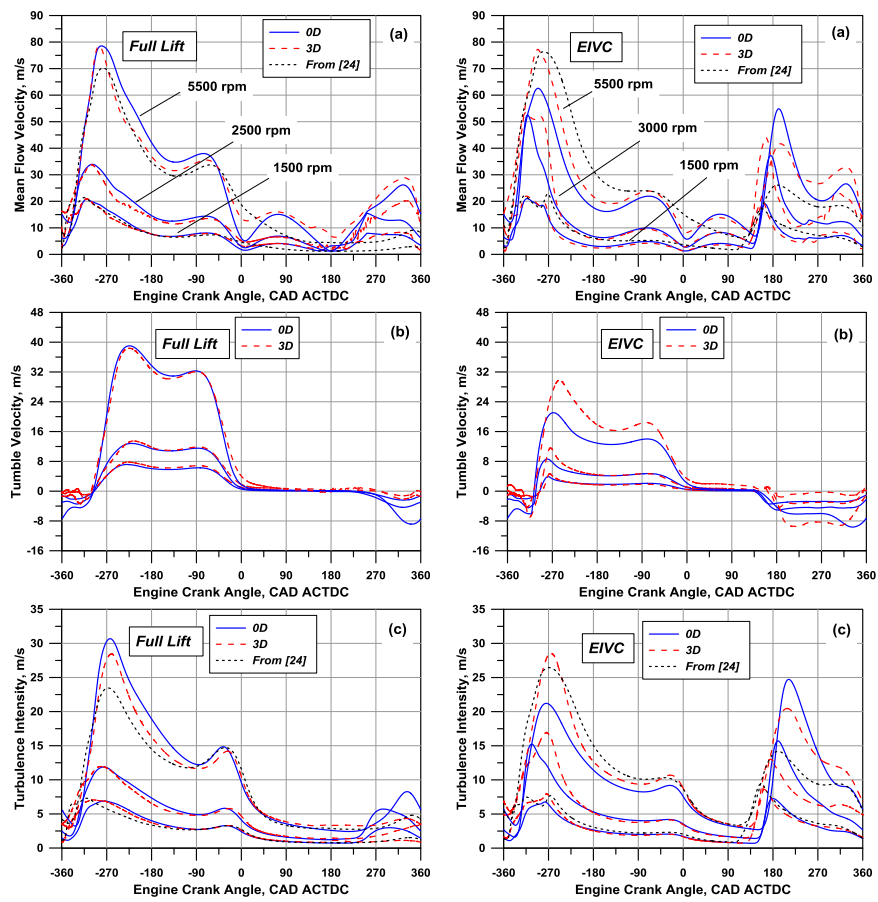

Figure 18. Model validation at various engine speeds. FL valve strategy.
Figure 19. Model validation at various engine speeds. EIVC valve strategy. 

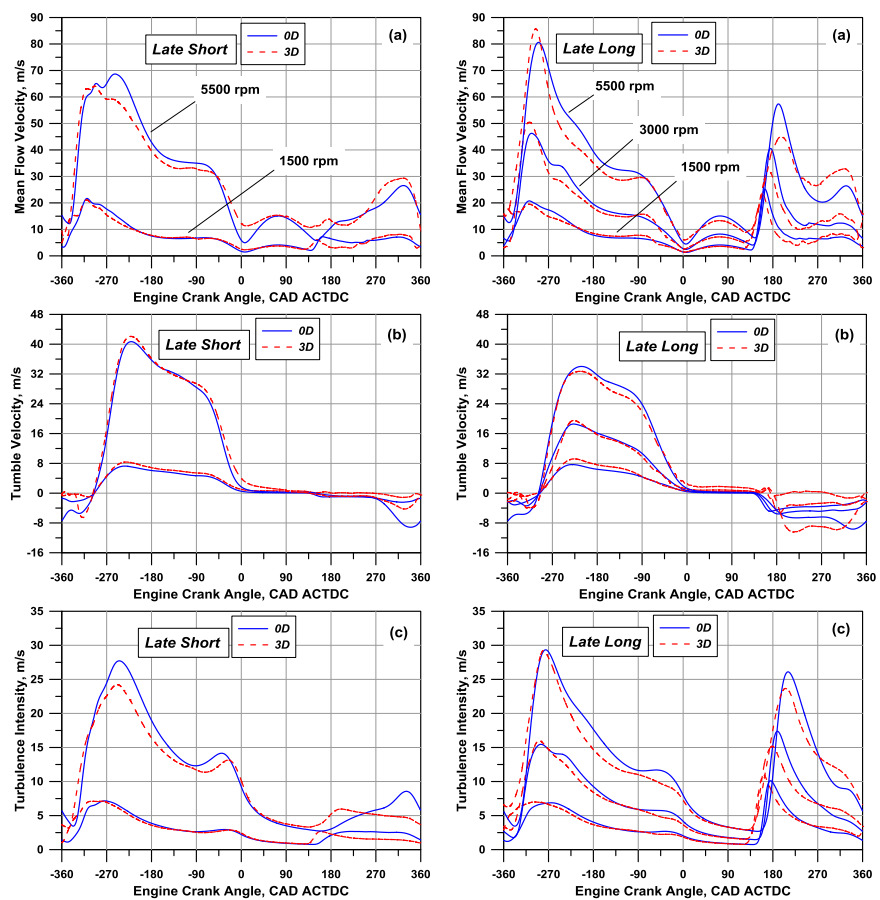

Figure 20. Model validation at various engine speeds. LIVC short valve strategy.
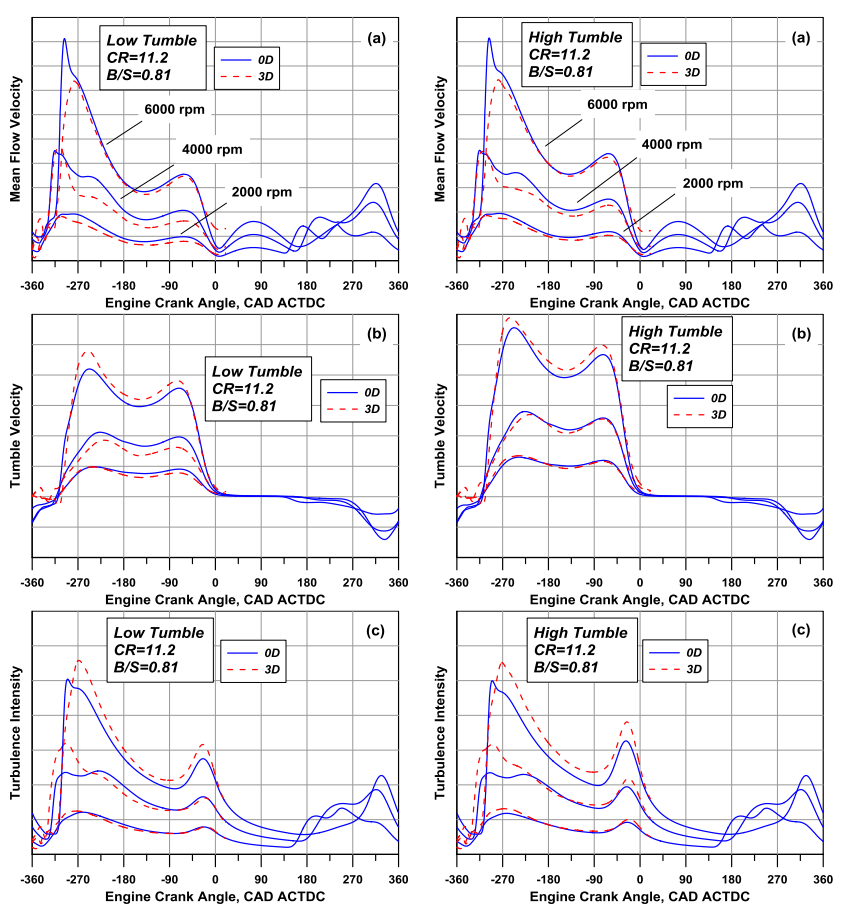

Figure 22. Model validation at various engine speeds. "Low Tumble" intake geometry.
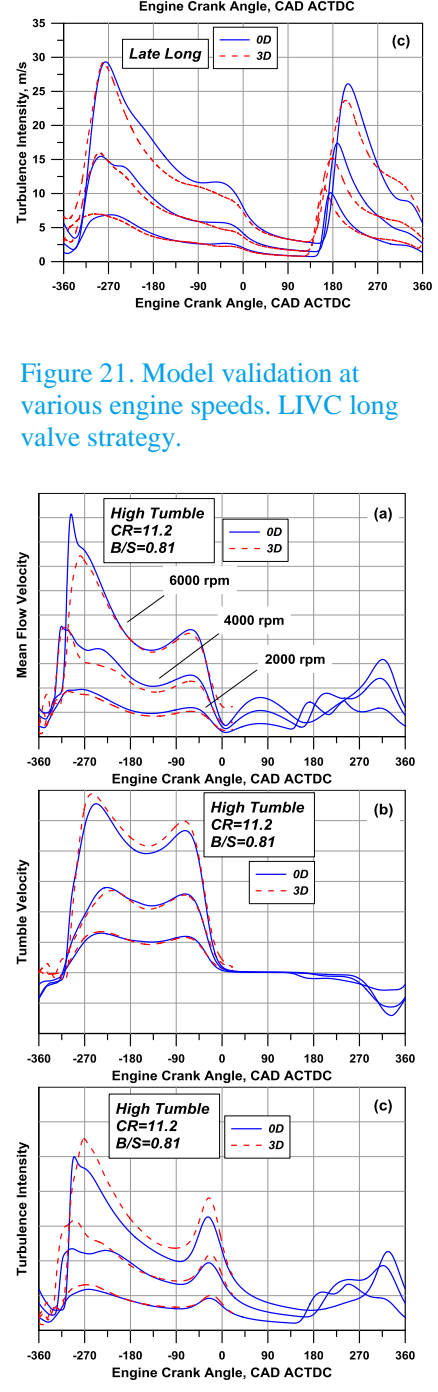

Figure 21. Model validation at various engine speeds. LIVC long valve strategy.

Figure 23. Model validation at various engine speeds. "High

Tumble" intake geometry.

Figure 18 and Figure 19 also show the comparisons of mean flow velocity and turbulence intensity with the results from the previous model version, proposed in [24]. The above assessment puts into evidence that the novel model has globally an accuracy similar to the previous version. Main improvements regard the description of the mean flow trend towards the end of the compression stroke, especially for the EIVC case, and during the expansion and exhaust

phases. This last advance is principally due to the piston motion handling expressed by eq. (22). Summarizing, the novel model capability in capturing the valve strategy effects on the in-cylinder flow and turbulence is slightly enhanced. Main developments concern the description of some geometrical engine characteristics, as discussed in the following.

\section{Intake port orientation analysis}

Figure 22 and Figure 23 report the comparison between "Low Tumble" and "High Tumble" configurations of the Engine B, differing in terms of intake port angle. A minimal re-tuning is required for the Engine B, starting from the constant set identified for the Engine A. New tuning is kept fixed for the two analyzed geometrical arrangements. Looking at the comparison between them, the procedure for the automatic flow coefficient definition seems reliable enough to get a good agreement with $3 \mathrm{D}$ data. The model exhibits the potential to correctly take into consideration the intake port angle effect on the velocity component effectively promoting the tumble $\left(v_{T}\right.$ in eq. (12)). Each monitored parameter follows the corresponding 3D trend and correctly scales with engine speed. Particularly, the turbulence speed-up is well reproduced for both tumble configurations. This behavior reveals a significant prospective in the proposed approach, since the latter allows to predict the turbulence intensity without any tumble coefficient measurement on a test bench at different port orientations. In this way, such a OD model can be also utilized to estimate burn rate variations due to runner inclination.

The effect description of the intake port inclination represents the main improvement compared to the previous model version [24]. The latter was not able to sense this geometrical parameter. This achievement mainly arises from the introduction of the tumble equation which, as said, considers the sole intake velocity component inducing the tumble motion development.
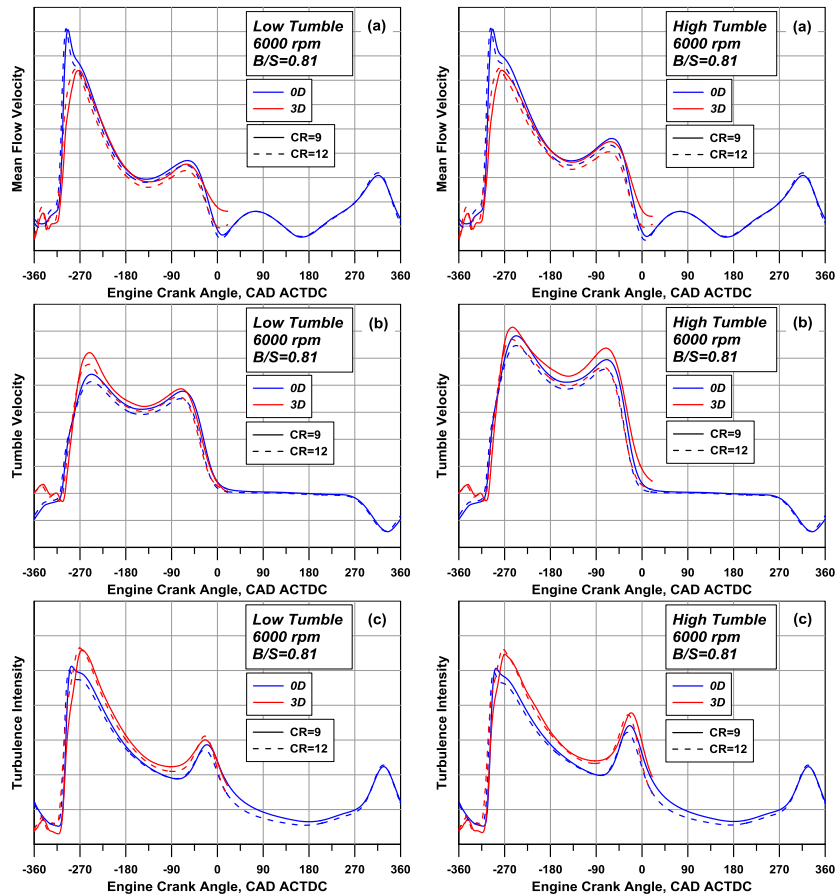

Figure 24. Model validation by varying compression ratio. "Low Tumble" intake geometry.
Figure 25. Model validation by varying compression ratio. "High Tumble" intake geometry.

Page 8 of 12 


\section{Compression ratio}

The tuning identified for the intake port analysis is also specified to investigate the effects of a modified compression ratio. Previous studies concern the base engine architecture, having a compression ratio of 11.2. In this section, a lower and a higher CR level are considered, namely, 9 and 12 .

The comparison between the two CRs is shown in Figure 24 and Figure 25, referring once again to the "Low Tumble" and "High Tumble" architectures, respectively. 3D findings show that the higher compression ratio involves a slightly reduced tumble level during the intake stroke, probably due to the more intense interaction between the incoming flow and the piston. In addition, the reduced combustion chamber height close to TDC is responsible for the advanced collapse. The above behavior is well reproduced by the $0 \mathrm{D}$ model. The relative trend variation is quite similar on both mean flow and tumble velocities, while minor differences occur on turbulence intensity. Similar considerations hold for both "Low Tumble" and "High Tumble" cases.

\section{Bore-to-stroke ratio}

The Engine B is geometrically "scaled" to design a high bore-tostroke configuration. The BS value of the base Engine B is 0.81 , while a $1.27 \mathrm{BS}$ ratio is defined for the scaled geometry. The results are reported in Figure 26 in terms of tumble velocities at 2000 and $6000 \mathrm{rpm}$. The tuning constants for the scaled engine required a little adjustment, mainly in terms of $c_{T i n 0}$ and $c_{f d 0}$ parameters. Results denote that an increased BS ratio turns in a reduced propensity to promote tumble, due to both increased piston interaction with intake flow, and wall shear during compression. The latter also determines an advanced tumble collapse. Tuning adjustment, although marginal, puts into evidence the need of further improving the model capability to define a more general decay function and a more reliable procedure for the tumble coefficient specification. An extra discussion on the tuning requirements will be given at the end of the Model Validation paragraph. Once adjusted, however, the model is able to correctly forecast the tumble behavior at various BS ratios.
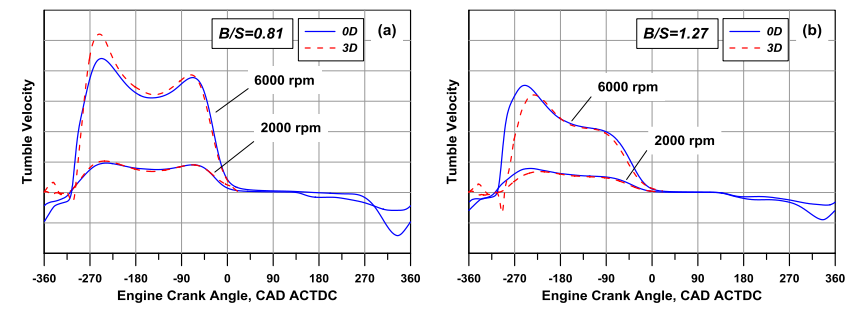

Figure 26. Model validation at various BS ratios. Tumble velocity.

\section{Two-valve engine}

A two-valve per cylinder engine is analyzed in this section, since this arrangement provides a very different evolution of the flow structure inside the cylinder. As pointed out in part I paper, tumble develops in 4 -valve architectures mainly around y-axis $\left(T_{y}\right)$. On the contrary, in 2-valve engines, ordered flow structures appear also around $\mathrm{x}$ - and $\mathrm{z}$ axis ( $T_{x}$ and $S_{z}$, respectively). More precisely, as shown in Figure 29 of part I, during the first part of the intake stroke, a $T_{y}$ is produced (Figure 27). Later, the incoming flow undergoes a deviation, due to the asymmetric valve arrangement, and $T_{x}$ and swirl motion, $S_{z}$, are also induced. Since eq. (5) describes the balance of a global tumble momentum, 3D data are post-processed to define an "equivalent" overall tumble level, $T_{x y}$, based on the following definition:

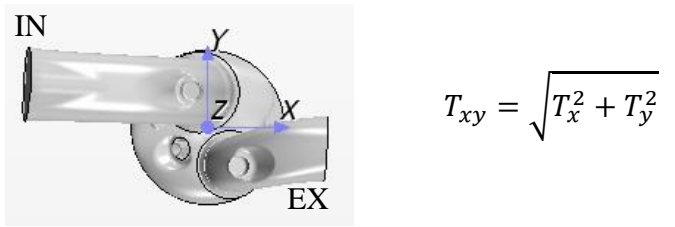

Figure 27. Two-valve arrangement.

Dashed red profiles in Figure 28a hence refer to the above definition. 3D tumble trends are surprisingly well reproduced by the 0D model. Particularly, a sharp increase of the tumble velocity approximately around $-250 \mathrm{CAD}$ at $6000 \mathrm{rpm}$ is detected by $0 \mathrm{D}$ and 3D results, as well. It corresponds to the crank angle where the incoming flow deviates, and a $T_{x}$ begins to develop. The model also well predicts the turbulence intensity, as shown in Figure 28b.
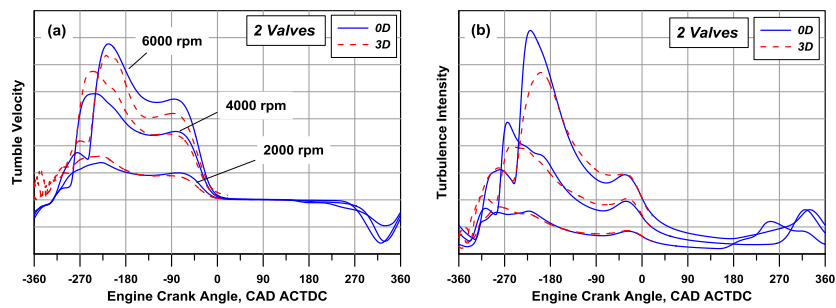

Figure 28. Model validation for a two-valve per cylinder engine. Tumble velocity (a) and Turbulence intensity (b)

\section{High-performance commercial engines}

In conclusion, two additional High-Performance Engines (HPEs), available in the in-series market, are considered. They differ in terms of each engine characteristics investigated above, such as valve phasing, port orientation, $\mathrm{CR}$, and $\mathrm{BS}$ ratio. OD model is tuned with reference to the $3 \mathrm{D}$ data for both engines, in order to appreciate the required variation of tuning constants. Despite of the relevant differences in terms of both geometrical parameters and operating conditions, a significant adjustment is only required for $c_{f d 0}$ constant, especially for HPE 1 (see Table 1). With the above adjustments a good agreement is once more obtained, as shown in Figure 29.

Table 1 finally summarizes the values of tuning constants utilized for each investigated engine arrangment. An advised default value is given for any parameter, together with the case-by-case applied multipliers. Parameter variation is quite limited and no adjustment was required for $c_{f d m}$ and $c_{P K k}$. Some additional refinement is, however, still recommended to further improve the model reliability and the engine-independence of tuning constants.
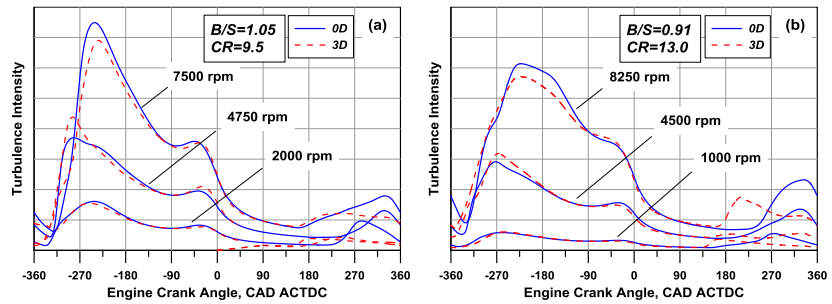

Figure 29. Model validation for high-performance engines. Turbulence intensity for HPE 1 (a) and HPE 2 (b). 
Table 1. Summary of engine arrangements and related tuning constants.

\begin{tabular}{|c|c|c|c|c|c|c|c|}
\hline \multicolumn{2}{|l|}{ Engine } & $\mathbf{A}$ & B & $\begin{array}{c}\text { B } \\
\text { Scaled } \\
\end{array}$ & \begin{tabular}{|c|} 
B \\
2-valve
\end{tabular} & HPE1 & HPE2 \\
\hline \multicolumn{2}{|l|}{ BS ratio } & 0.94 & 0.81 & 1.27 & 0.81 & 1.05 & 0.91 \\
\hline \multicolumn{2}{|l|}{ CR } & 9.9 & $9-12$ & 9.0 & 11.2 & 9.5 & 13.0 \\
\hline \multicolumn{2}{|l|}{ Tumble } & High & $\begin{array}{l}\text { Low- } \\
\text { High } \\
\end{array}$ & Low & Low & Medium & Low \\
\hline \multicolumn{2}{|c|}{ Valve per cyl. } & 4 & 4 & 4 & 2 & 4 & 4 \\
\hline \multicolumn{2}{|c|}{ Valve control } & VVA & Fixed & Fixed & Fixed & VVT & VVT \\
\hline Constant & Def. & \multicolumn{6}{|c|}{ Multiplier } \\
\hline$c_{\text {Kino }}$ & 0.86 & 0.87 & 1.16 & 1.16 & 0.87 & 0.93 & 0.87 \\
\hline$c_{\text {Tino }}$ & 0.88 & 0.85 & 1.02 & 0.85 & 1.13 & 1.05 & 1.08 \\
\hline$c_{f d 0}$ & 0.22 & 0.96 & 0.92 & 1.01 & 1.37 & 0.73 & 1.10 \\
\hline$c_{f d m}$ & 0.90 & 1.00 & 1.00 & 1.00 & 1.00 & 1.00 & 1.00 \\
\hline$c_{P K k}$ & 2.50 & 1.00 & 1.00 & 1.00 & 1.00 & 1.00 & 1.00 \\
\hline
\end{tabular}

\section{Conclusions}

In the present work, a 0D model of turbulence, mean flow and tumble is developed, based on the results of extensive 3D analyses presented in the companion part I paper.

The model is described in detail, proposing both a 3-eq. version and a more complex 4-eq. version. The latter, however, does not seem to improve the accuracy, mainly due to the difficulties in describing, in a $0 \mathrm{D}$ environment, the $3 \mathrm{D}$ distribution of the dissipation rate. In fact, the latter exhibits a great not-uniformity within the cylinder, with values orders of magnitude higher near to the intake and exhaust valves, especially in case of flow reversals. The 3-eq. concept is hence preferred.

Subsequently, a parametric analysis describing the role of each tuning constant is presented, with the aim of advising a tuning procedure. Once tuned, the 3-eq. model is extensively tested for different engines, and in various operating conditions.

In particular, EIVC, FL and LIVC valve strategies can be handled with a single set of tuning constants. In each case, a satisfactory agreement on various turbulence and mean flow parameters is demonstrated.

The second part of the paper is focused on the model potential assessment in correctly describing variations in some geometrical parameters. Different inclinations of the intake port are analyzed, to foresee the in-cylinder tumble motion development and turbulence evolution during the intake and the compression strokes. Variations of CR, BS ratio and valve arrangement (both 4-valve and 2-valve per cylinder) are also studied. In most cases, a very good agreement with 3D data is obtained, with reduced or null modifications of tuning constants.

Finally, two high-performance engines are considered, summing up all the effects of the above analyzed parameters.

Reliability and consistency of the proposed model is proven to be high, although some additional improvement is advised, mainly concerning the definition of proper tumble flow coefficient. Activities are currently on going to this aim, once again utilizing 3D models. The latter are used to characterize physical and geometrical effects, to be later synthesized in the phenomenological model, thus following a hierarchical 1D-3D integration.

\section{References}

1. Hires, S., Tabaczynski, R., Novak, J., "The Prediction of Ignition Delay and Combustion Intervals for a Homogeneous Charge, Spark Ignition Engine", SAE Technical Paper 780232, 1978, doi: $10.4271 / 780232$.

2. Blizard, N. Keck, J., "Experimental and Theoretical Investigation of Turbulent Burning Model for Internal Combustion Engines", SAE Technical Paper 740191, 1974, doi: 10.4271/740191.

3. Morel, T., Rackmil, C., Keribar, R., and Jennings, M., "Model for Heat Transfer and Combustion In Spark Ignited Engines and its Comparison with Experiments", SAE Technical Paper 880198, 1988, doi: $10.4271 / 880198$.

4. Richard, S., Bougrine, S., Font, G., Lafossas, F.A., Le Berr, F. "On the Reduction of a 3D CFD Combustion Model to Build a Physical 0D Model for Simulating Heat Release, Knock and Pollutants in SI Engines", Oil \& Gas Science and TechnologyRev. IFP. 64(3): 223-242, 2009. doi: 10.2516/ogst/2008055.

5. Gatowsky, J., Heywood, J., "Flame Photographs in a SparkIgnition Engine", Combustion and Flame. 56(1):71-81, 1984, doi: $10.1016 / 0010-2180(84) 90006-3$.

6. Gouldin, F. "An application of Fractals to Modeling Premixed Turbulent Flames", Combustion and Flame. 68(3):249-266, 1987, doi: 10.1016/0010-2180(87)90003-4.

7. Millo, F., Luisi, S., Borean, F., Stroppiana, A., "Numerical and experimental investigation on combustion characteristics of a spark ignition engine with an early intake valve closing load control", Fuel 121: 298-310, 2014, doi: 10.1016/j.fuel.2013.12.047.

8. Luo, X., Teng, H., Lin, Y., Li, B. et al., "A Comparative Study on Influence of EIVC and LIVC on Fuel Economy of a TGDI Engine Part II: Influences of Intake Event and Intake Valve Closing Timing on the Cylinder Charge Motion", SAE Technical Paper 2017-01-2246, 2017, doi: 10.4271/2017-01$\underline{2246}$.

9. Borgnakke, C., Arpaci, V., Tabaczynski, R., “A Model for the Instantaneous Heat Transfer and Turbulence in a Spark Ignition Engine”, SAE Technical Paper 800287, 1980, doi: 10.4271/800287.

10. Morel, T., Mansour, N., "Modeling of Turbulence in Internal Combustion Engines", SAE Technical Paper 820040, 1982, doi: 10.4271/820040.

11. Morel, T. Keribar, R., "A Model for Predicting Spatially and Time Resolved Convective Heat Transfer in Bowl-in-Piston Combustion Chambers", SAE Technical Paper 850204, 1985, doi: $10.4271 / 850204$.

12. Sjeric, M., Kozarac, D., Bogensperger, M. "Implementation of a Single Zone k- $\varepsilon$ Turbulence Model in a Multi Zone Combustion Model”, SAE Technical Paper 2012-01-0130, 2012, doi: 10.4271/2012-01-0130.

13. Dulbecco, A., Richard, S., Laget, O., Aubret, P., "Development of a Quasi-Dimensional K-k Turbulence Model for Direct Injection Spark Ignition (DISI) Engines Based on the Formal Reduction of a 3D CFD Approach", SAE Technical Paper 201601-2229, 2016, doi: 10.4271/2016-01-2229.

14. Kim, N., Kim, J., Ko, I., Choi, H. et al., "A Study on the Refinement of Turbulence Intensity Prediction for the Estimation of In-Cylinder Pressure in a Spark-Ignited Engine", SAE Technical Paper 2017-01-0525, 2017, doi: 10.4271/201701-0525.

15. Lafossas, F., Colin, O., Le Berr, F., Menegazzi, P., "Application of a New 1D Combustion Model to Gasoline Transient Engine Operation", SAE Technical Paper 2005-01-2107, 2005, doi: $\underline{10.4271 / 2005-01-2107 .}$. 
16. Ramajo, D., Zanotti, A., Nigro, N., "Assessment of a zerodimensional model of tumble in four-valve high performance engine", International Journal of Numerical Methods for Heat \& Fluid Flow 17(8): 770-787, 2007, doi: 10.1108/09615530710825765.

17. Achuth, M., Mehta, P.S. "Predictions of tumble and turbulence in four-valve pentroof spark ignition engines", International Journal of Engine Research 2(3): 209-227, 2001, doi: $\underline{10.1243 / 1468087011545442 .}$.

18. Grasreiner, S., Neumann, J., Luttermann, C., Wensing, M., Hasse, C., "A quasi-dimensional model of turbulence and global charge motion for spark ignition engines with fully variable valvetrains", International Journal of Engine Research 15(7): 805 - 816, 2014, doi: 10.1177/1468087414521615.

19. Fogla, N., Bybee, M., Mirzaeian, M., Millo, F. et al., "Development of a K-k- $\in$ Phenomenological Model to Predict In-Cylinder Turbulence", SAE Int. J. Engines 10(2):562-575, 2017, doi: 10.4271/2017-01-0542.

20. Dai, W., Newman, C., Davis, G., "Predictions of In-Cylinder Tumble Flow and Combustion in SI Engines with a QuasiDimensional Model", SAE Technical Paper 961962, 1996, doi: $\underline{10.4271 / 961962 .}$.

21. Jones, P. Junday, J., "Full Cycle Computational Fluid Dynamics Calculations in a Motored Four Valve Pent Roof Combustion Chamber and Comparison with Experiment", SAE Technical Paper 950286, 1995, doi: 10.4271/950286.

22. Khalighi, B., El Tahry, S., Haworth, D., Huebler, M., "Computation and Measurement of Flow and Combustion in a Four-Valve Engine with Intake Variations", SAE Technical Paper 950287, 1995, doi: 10.4271/950287.

23. Bozza, F., De Bellis, V., Berni, F., D’Adamo, A., Maresca, L., "Refinement of a 0D Turbulence Model to Predict Tumble and Turbulent Intensity in SI Engines. Part I: 3D Analyses", SAE Technical Paper 2018-01-0850, 2018, doi: 10.4271/2018-01$\underline{0850}$.

24. De Bellis, V., Bozza, F., Fontanesi, S., Severi, E. et al., "Development of a Phenomenological Turbulence Model through a Hierarchical 1D/3D Approach Applied to a VVA Turbocharged Engine", SAE Int. J. Engines 9(1):506-519, 2016, doi: $\underline{10.4271 / 2016-01-0545}$.

\section{Contact Information}

F. Bozza, Full Professor, (fabio.bozza@unina.it),

L. Teodosio, Research Fellow, (luigi.teodosio@unina.it),

V. De Bellis, Researcher, (vincenzo.debellis@unina.it),

University of Naples “Federico II", Naples, Italy, +39-081-7683274.

S. Fontanesi, Assistant Professor, (stefano.fontanesi@ unimore.it), University of Modena and Reggio Emilia, Modena, Italy, +39- 059 2056114.

\section{Acronyms}

$\begin{array}{ll}\text { 0D/1D/3D } & \text { Zero/One/Three-Dimensional } \\ \text { ACTDC } & \text { After compression top dead center } \\ \text { BS } & \text { Bore-to-Stroke ratio } \\ \text { CAD } & \text { Crank angle degree } \\ \text { CFD } & \text { Computational fluid dynamics } \\ \text { CR } & \text { Compression ratio } \\ \text { EIVC } & \text { Early intake valve closing } \\ \text { FL } & \text { Full lift } \\ \text { HPE } & \text { High performance engine } \\ \text { ICE } & \text { Internal combustion engine } \\ \text { LIVC } & \text { Late intake valve closing } \\ \text { RNG } & \text { Renormalization group } \\ \text { SI } & \text { Spark ignition } \\ \text { Page 11 } 12 & \end{array}$

Top dead center

Variable valve actuation

Variable valve timing

\section{Symbol}

\begin{tabular}{|c|c|}
\hline $\boldsymbol{B}$ & Bore \\
\hline$c_{f d 0,} c_{f d m}$ & Tuning constants of tumble decay function \\
\hline$c_{\text {Kino }}$ & Tuning constant of inlet flow coefficient \\
\hline$c_{P K k}$ & Tuning constant of turbulence Production \\
\hline$c_{r T 0,} c_{r T m}$ & Parameters for tumble radius adjustment \\
\hline$c_{\text {Tino }}$ & Tuning constant of tumble flow coefficient \\
\hline$f_{d}$ & Decay function of tumble \\
\hline$g_{i}$ & Gravity acceleration along i-th-axis \\
\hline $\boldsymbol{H}$ & Piston position referred to cylinder head \\
\hline $\boldsymbol{k}$ & Turbulent kinetic energy \\
\hline $\boldsymbol{K}$ & Mean flow kinetic energy \\
\hline$K_{\text {pist }}$ & Kinetic energy related to piston motion \\
\hline$K_{T}$ & Kinetic energy related to tumble motion \\
\hline$L_{I}$ & Integral length scale \\
\hline$m$ & Mass \\
\hline$r_{T}$ & Tumble radius \\
\hline$S, S_{i j}$ & Strain tensor \\
\hline$S_{z}$ & Swirl momentum (referred to z-axis) \\
\hline$t$ & Time \\
\hline$t_{T}$ & Characteristic time scale of tumble \\
\hline$T$ & Tumble momentum \\
\hline$T_{x}, T_{y}, T_{x y}$ & $\begin{array}{l}\text { Tumble momentum referred to } \mathrm{x} \text { - and } \mathrm{y} \text {-axes, and } \\
\text { their composition }\end{array}$ \\
\hline$u_{i}$ & Velocity components along i-th axis \\
\hline$u^{\prime}$ & Turbulence intensity \\
\hline$u_{\text {pist }}$ & Instantaneous piston velocity \\
\hline$U_{f}, U_{f K}$ & $\begin{array}{l}\text { Mean flow velocities, with and without contribution } \\
\text { from piston motion }\end{array}$ \\
\hline$U_{T}$ & Tumble velocity \\
\hline$v$ & Flow velocity throughout the valve \\
\hline$x, y, z$ & Cartesian coordinates \\
\hline$x_{i}$ & Cartesian coordinate along i-th axis \\
\hline
\end{tabular}

\section{Greeks}

$\alpha$
$\varepsilon$
$\eta$
$v_{t}$
$\rho$
$c_{\varepsilon i,} c_{\mu}, \eta 0, \beta$
$\Sigma_{c y l}$

\section{Pedices}

Intake port angle

Dissipation rate

Parameter in $k$ - $\varepsilon$ RNG model

Turbulent viscosity

Density

$k-\varepsilon$ RNG turbulence model constants

Summation over all cylinder cells

Page 11 of 12 


\section{Apices}

Temporal derivative

Page 12 of 12 